\title{
Assessing the Accuracy of Soot Nanoparticle Morphology Measurements Using Three-Dimensional Electron Tomography
}

\author{
Ephraim Haffner-Staton, Antonino La Rocca, Alasdair Cairns, Michael McGhee, and Mike Fay
}

University of Nottingham

Citation: Haffner-Staton, E., La Rocca, A., Cairns, A., McGhee, M. et al., "Assessing the Accuracy of Soot Nanoparticle Morphology Measurements Using Three-Dimensional Electron Tomography," SAE Technical Paper 2019-01-1188, 2019, doi:10.4271/2019-01-1188.

\section{Abstract}

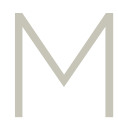

orphology plays an important role in determining behaviour and impact of soot nanoparticles, including effect on human health, atmospheric optical properties, contribution to engine wear, and role in marine ecology. However, its nanoscopic size has limited the ability to directly measure useful morphological parameters such as surface area and effective volume. Recently, 3D morphology characterization of soot nanoparticles via electron tomography has been the subject of several introductory studies. So-called '3D-TEM' has been posited as an improvement over traditional 2D-TEM characterization due to the elimination of the error-inducing information gap that exists between 3-dimensional soot structures and 2-dimensional TEM projections. Little follow-up work has been performed due to difficulties with developing methodologies into robust high-throughput techniques. Recent work by the authors has exhibited significant improvements in efficiency, though as yet due consideration has not been given to assessing fidelity of the technique. This is vital to confirm significant and tangible improvements in soot-characterization accuracy that will establish 3D-TEM as a legitimate tool. Synthetic ground-truth data was developed to closely mimic real soot structures and the 3D-TEM volume-reconstruction process. A variety of procedures were tested to assess the magnitude and nuances of deviations from ground-truth values. Results showed average $\mathrm{Z}$-elongation due to the 'missing-wedge' at 3.5\% for the previously developed optimized procedure. Mean deviations from ground-truth in volume and surface area were $2.0 \%$ and $-0.1 \%$ respectively. Results indicate highly accurate 3D-reconstruction can be achieved with an optimized procedure that can bridge the gap to permit highthroughput 3D morphology characterization of soot.

\section{Introduction}

oot nanoparticles emitted from automotive engines are an extremely important pollutant due to their wide and varied effects in the environment. These include immune and inflammatory effects when inhaled $[\underline{1}, \underline{2}, \underline{3}]$, effect on the balance of microbial processes in marine environments $[\underline{4}, \underline{5}, \underline{6}]$, contribution to the greenhouse effect as an atmospheric species $[\underline{7}, \underline{8}]$, and effects on automotive internal combustion engine (ICE) efficiency via lubricant oil entrainment [9]. Key to the development of strategies for the reduction of soot emissions, or for its capture/storage/removal is understanding the processes governing soot formation and oxidation. Research in this area includes characterization of fundamental chemical and physical properties of soot such as elemental composition [10], degree of graphitization [11], surface functional group chemistry [12], oxidative reactivity $[13, \underline{14}]$, carbon nanostructure $[\underline{15}, \underline{16}]$, surface area $[\underline{10}, \underline{17}]$, fractal geometry [18], and size [19].
Morphological characterization is particularly important due to the defining role properties such as fractal geometry and surface area play in determining effects such as depth of penetration of soot into the lungs [1], lifetime and radiative potential as a atmospheric aerosol $[\underline{7}, \underline{18}]$, and contribution to wear and viscosity increases when entrained within engine lubrication oils $[\underline{9}, \underline{20}]$. Accurate characterization of such parameters is important for understanding the causes of such behavior, e.g. when incorporated into studies modelling soot behavior $[\underline{21}, \underline{22}, \underline{23}]$.

Soot nanoparticles are formed from the random aggregation of spherical carbonaceous sub-units known as primary particles that results in characteristic structures that are complex, 3-dimensional, and fractal-like. Primary particles are usually in the size range of $10-50 \mathrm{~nm}$ in diameter [24] and nanoparticles from automotive engines are predominantly $<200 \mathrm{~nm}$ in size [25], though larger aggregates may form depending on the source and experimental sampling method $[26,27]$. Soot's complex 3D structure is considered to be mass-fractal, i.e. possessing a

(c) 2019 The Authors. Published by SAE International. This Open Access article is published under the terms of the Creative Commons Attribution License (http:// creativecommons.org/licenses/by/4.0/), which permits distribution, and reproduction in any medium, provided that the original author(s) and the source are credited. 
self-similar pattern (e.g. a snowflake), but one that only repeats to a limited degree $[\underline{28}, \underline{29}]$. Primary particles possess a distinctive core-shell structure, as can be observed by TEM [ $\underline{30}]$. The inner core is up to $10 \mathrm{~nm}$ in diameter, comprising of spherical fullerenoid structures around $1-2 \mathrm{~nm}$ in diameter surrounded by relatively amorphous turbostratic carbon. This inner core is surrounded by a shell-like layered structure of short graphitic micro-crystallites that are $\sim 3 \mathrm{~nm}$ in width. In some cases, e.g. soot from gasoline direct injection (GDI) engines, an additional outer 'dusting' of predominantly amorphous carbon may be seen $[31,32]$. On occasion other unusual features have been observed, such as hollow primary particles for soot collected on diesel particulate filters [33].

Characterization of soot morphology can be carried out using a variety of techniques ranging in terms of complexity and experimental difficulty, speed and sample size, and level of detail and accuracy of measurements. Different methods may need to be employed depending on the way soot is produced and sampled. In many cases, soot can be sampled and measured directly from the engine exhaust stream via methods employing electrical mobility measurements [푼 $\underline{35}]$. These methods measure particle size distributions and number concentrations by charging aerosol particles collected from the exhaust stream and measuring their movement through a medium in response to an applied electrical field. Similarly, size distribution and number concentration measurements for the hydrodynamic diameter of soot nanoparticles in solution can be obtained through light scattering techniques . Dynamic light scattering (DLS) and Nanoparticle Tracking Analysis (NTA) are two such methods that have been used to study flame-generated soot suspended in organic solvents [주], and engine soot entrained within lubricant oils [37]. Tracked Rayleigh scattering of laser light by soot nanoparticles is used to infer sizes, and for NTA size distributions are built from measurements of individual particles. While these methods can be performed rapidly and results are based on statistically significant numbers of measurements, the resulting electrical mobility or hydrodynamic diameters are equivalent to the spherical space occupied by nanoparticles tumbling \& rotating either in solution or the gas phase. As such, it provides relatively little information on the complex, fractal morphology known to be characteristic of soot nanoparticles.

For more detailed characterization direct observation of the nanoparticle morphology is required. Due to the nanoscopic size of soot this requires the use of high magnification techniques, with a majority of investigations using transmission electron microscopy (TEM) [르, $\underline{38}, \underline{39}, \underline{40}, \underline{41}, \underline{42}, \underline{43}]$, and to a lesser extent scanning electron microscopy (SEM) [44, 45]. TEM and SEM produce 2-dimensional projections of soot nanoparticles, as shown in Figure 1. As soot is predominantly carbon, nanoparticles appear low contrast and semi-transparent to the electron beam. This transparency allows some appreciation of the 3-dimensional nature of soot nanoparticles, though fundamentally only 2D measurements can be made directly. Typically, image-processing software such as ImageJ/Fiji is used to select pixels in the image that pertain to the nanoparticles [46]. Parameters such as projected area (in $\mathrm{nm}^{2}$ ), mean primary particle diameter, maximum length, skeleton length, radius of gyration, circularity, fractal dimension (purely 2D), etc. can then be measured. These results are then used to infer the values
FIGURE 1 Example of a 2D-TEM projection of a sootin-oil nanoparticle

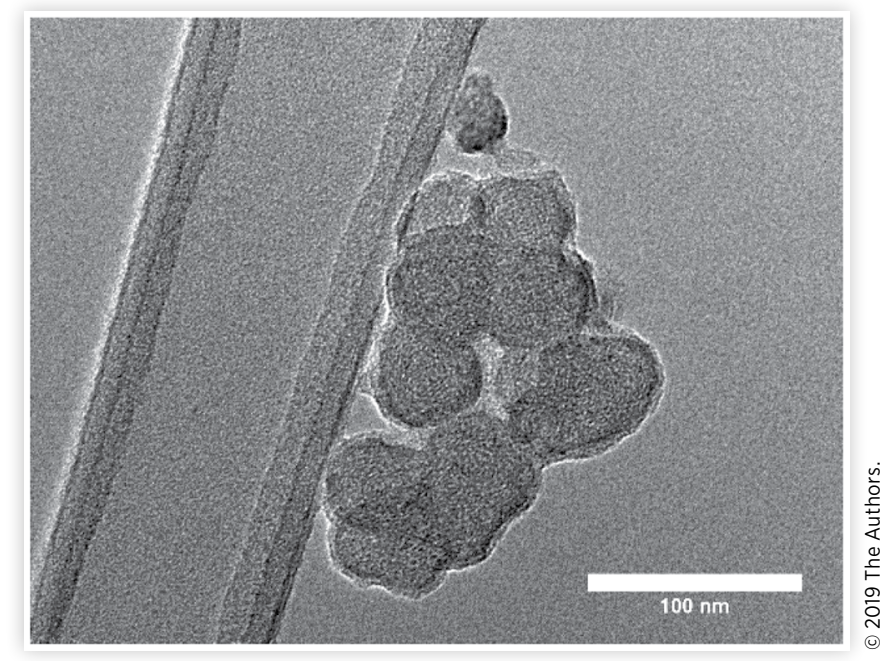

of 3D properties such as volume, surface area, 3D fractal dimension etc. This information gap that exists between true 3D morphology of soot, and the 2D morphology that is available is the primary source of uncertainty in 2D-TEM measurements.

In many cases, an average primary particle diameter is first determined manually. The number of primary particles comprising the whole nanoparticle is then estimated using the following equation $[\underline{29}, \underline{47}]$, derived from the power-law relationship [요]:

$$
N_{p}=k_{a}\left(A_{e f f} / A_{p p}\right)^{\alpha}
$$

Where $A_{\text {eff }}$ is the projected area of the entire nanoparticle, $A_{p p}$ is the circular projected area of an assumed perfectly spherical primary particle, of the measured average diameter. The values of prefactor $k_{a}$ and exponent $\alpha$ were determined using a combination of experimental and simulated data, and account for the overlap of primary particles, and the unseen 3D extent of the particle. Values of $\mathrm{k}_{\mathrm{a}}=1.15$ and $\alpha=1.09$ were empirically determined by Köylü et al. [29], using a combination of simulated aggregates created to match experimental fractal dimension values, and soot sampled from diffusion flames of various fuels. Problems with empirically determined correction factors such as these can arise when they are applied to soot samples that differ greatly in their nature of formation, as may be the case for diesel and gasoline ICE soot. For example, Köylü's work included contributions from particles with large numbers of primary particles (500-1000), whereas recent work by Martos suggested that only a small fraction (4.5\%) of aggregates from diesel exhaust are comprised of $>200$ primary particles [25]. In some cases, a variety of short cuts and assumptions have to be used in order to extract 3D parameters. For example, from calculated values of $N_{p}$ (using equation 1), nanoparticle volume may be estimated by simply multiplying by the volume a single primary particle [49], ignoring any overlap of particles and assuming all primary particles to be the same size and perfectly spherical. In some cases, fractal dimension determined using $2 \mathrm{D}$ methods is simply assumed to be equivalent to the $3 \mathrm{D}-\mathrm{D}_{\mathrm{f}}$ $[\underline{50}, \underline{51}]$, despite inherent 2D limitations. 
Work considering real and simulated 3D soot data has routinely observed large deviations between 3D- and 2D-derived morphological characterizations. While Rogak's early work on simulated soot particles culminated in an estimated $10-20 \%$ underestimation by 2D-methods [28], more recent work on 3D-TEM of soot nanoparticles found differences in volume and surface area of significantly larger magnitude [ $\underline{52}, \underline{53}]$. Martos' recent study of simulated aggregates observed that radius of gyration from $2 \mathrm{D}$ methods was underestimated by as much as $45 \%$, and was even more pronounced for larger particles [25].

Another source of uncertainty in 2D-TEM measurements derives from the fact that the appearance of a fractal soot nanoparticle in $2 \mathrm{D}$ can vary greatly depending upon the orientation in which it appears on the TEM grid. This has been noted by a number of researchers $[\underline{18}, \underline{28}, \underline{52}, \underline{54}]$, though has received relatively little consideration in the majority of studies of soot morphology. Our own work observed variation in projected area by as much as $60 \%$ over a $\pm 60^{\circ}$ orientation range [53], and results of similar magnitude were observed by Adachi et al. [18]. Fundamentally, 2D-3D correction factors cannot account for the effect of this 'orientation uncertainty' in 2D-TEM. Not one single orientation is any more significant than any other, and static values of correction factors cannot account for $>1$ orientation for each particle. Even if an ideal set of correction factors/equations were developed (i.e. robustly developed using a large number of particles for the exact sample under study), it would still only be able to transform $2 \mathrm{D}$-measured values to within a region of uncertainty around the exact $3 \mathrm{D}$ values. The size of the 'cloud' of uncertainty is directly related to the degree of variation in the appearance of the particle in different orientations in 2D-TEM, and as mentioned this has been seen to be significant. Therefore, even if excellent correction factors are employed, there is still an extra statistical 'noise' around 2D-derived 3D morphological results. As the end goal of the majority of these types of studies is to arrive at some average/characteristic values that describe the soot nanoparticles of a particular sample, the result is to significantly increase the number of $2 \mathrm{D}$-derived results that are needed to overcome the effect of the orientation uncertainty and produce statistically relevant values.

The sources of error and uncertainty mentioned for 2D-TEM are not mutually exclusive, but can reinforce one another. Measurements via 2D-TEM may use unsuitable correction factors as well as underappreciate the contribution of alternate 2D-projections, resulting in under/over-estimations $v s$. real $3 \mathrm{D}$-derived values. The consequences of such compound errors could be severe, and surely play in a role in the limited understanding of numerous soot-related phenomena. For example, the effect of soot on engine lubricant oil is poorly understood (in a holistic sense) despite 5 decades of research in the area. Shortcomings in characterization methodologies are of direct concern, as noted by researchers on several occasions when more limited descriptions of soot (e.g. mass fraction in oil samples) are used to try and correlate with observed effects such as increases to viscosity and wear in engines. Bardasz et al. even explicitly mentioned that advances in the characterization of particle shape were likely to improve the understanding of the relationship between soot and oil viscosity increase [55]. The magnitude and undefined nature of the error associated with 2D-TEM characterizations poses a significant barrier to the understanding of soot-related phenomena in a variety of disciplines. Development of reliable 3D-based characterization technology could offer significant improvements in this regard.

\section{Electron Tomography for Soot Nanoparticles}

Overall there are myriad sources of uncertainty to affect 2D-derived results that are unavoidable when using 2D-TEM. If it were possible to accurately recreate particle volume in a timely manner, 3D morphological parameters could be extracted directly without the need for correction factors and approximations, and avoiding issues such as the orientation of the particle. Potentially this could result in much more accurate characterization. As morphology plays such an important role in determining the behavior of soot and thus in developing models for soot behavior, it is imperative that characterization results are as accurate as possible. The recreation of soot nanoparticle volume in $3 \mathrm{D}$ is possible via tomographic reconstruction using TEM (sometimes known as 3D-TEM), as has been exhibited in several introductory studies $[\underline{18}, \underline{52}, \underline{53}, \underline{56}, \underline{57}]$. However, to date there has been relatively little follow-up due to the technique being far too slow to compete with 2D-TEM despite potentially significant improvements in quality.

Fourier Slice Theorem describes the underlying principles of tomographic reconstruction [58]. Simply put, all the information that is needed to recreate an object is $3 \mathrm{D}$ is contained within the full set of projections of the object in 2D. Another important prerequisite of tomographic reconstruction is the Projection Requirement; i.e. that the object to be reconstructed is partially transparent in the projection images, and that the relationship between transparency and object density is monotonic [59]. For soot this means that in TEM images the grey-level of the particle is darker in areas where there is more matter (e.g. multiple particles overlapping), and vice versa; and this requirement is fulfilled. By contrast, this method of electron tomography would not be suitable for reconstruction of metal nanoparticle volumes, for example, as they are opaque to the electron beam.

The most applicable way in which to obtain the set of 2D projections for soot is to use TEM equipped with a high-tilt specimen holder. This allows the sample stage to be rotated, so that images of the same nanoparticle can be acquired from different viewing angles. In practice, the TEM stage is tilted in small increments $\left(1-5^{\circ}\right)$ over a tilt range defined by the operator. Due to mechanical limitations of the TEM equipment, the maximum tilt range that is typically achievable is $\pm 60-70^{\circ}$. A so-called 'tilt-series' can comprise as many as 100-200 images depending on the tilt range and increment. It should be noted that a $\pm 60-70$ tilt range clearly does not constitute a full set of projections as is stipulated in Fourier Slice Theorem.

Reconstruction is the computational process by which $2 \mathrm{D}$ information in the form of the tilt-series is used to recreate the $3 \mathrm{D}$ volume in the form of a tomogram. In Fourier Slice Theorem, a 2D Fourier transform (FT) of the 2D projection of the nanoparticle at angle $\theta$ (tilt-angle of the TEM stage) is 
exactly equal to a line through the 3D Fourier representation of the original 3D object. This line passes through the origin at the angle $\theta$, and thus the 2D FT of an entire tilt-series could be used to recreate the 3D Fourier representation of the model. The original model would then be recreated by an inverse FT on this combined data. In practice, this method is too difficult to carry out, and instead a wide variety of different algorithms exist to approximate this situation [으]. Algorithms vary depending on the needs of researcher, and in many cases very advanced methods can be used to reconstruct volumes in high detail. Such methods are computationally slow, and often require additional inputs of the designations of discrete grey levels to particular components of a tomogram $[61,62]$. These particular methods are not applicable to soot as this information does not exist. However, there do remain a number of open-source methods that vary in their speed and quality. The most simplistic method is weighted back-projection (WBP), which approximates the Fourier approach in realspace by projecting pixel densities from the tilt series through an empty 3D volume [60]. In TEM images darker pixels refer to particle matter, and in the tomogram these darker pixels reinforce one another to designate the position of particle matter in 3D. Another widely used method is the simultaneous iterative reconstruction technique (SIRT) [60], which creates estimated tomograms from which it works in reverse to recreate TEM projections. The estimated tomograms are then fine-tuned with each iteration so that the recreated $2 \mathrm{D}$ projections match the real TEM images more closely. In the limit of infinite iterations, the SIRT tomogram is exactly equal to the original object. Both have been used to study soot nanoparticles $[\underline{18}, \underline{53}, \underline{63}, \underline{64}]$.

Reconstruction creates 3D information of the original structure in the form of a tomogram. As the tilt-series contains pixels that correspond to the background TEM support film, this information is carried through the reconstruction. A tomogram is solid $3 \mathrm{D}$ volume of voxels, where the colour value of the voxels is proportional to the density of matter at that position (e.g. particles are dark against a lighter background). In order to produce a model of the reconstructed particle, the voxels pertaining to the structure of interest must be distinguished from those of the background; this is known as segmentation. Segmentation of a 3D tomogram is usually carried out by arranging the tomogram into 1 voxel thick slices in the XY-plane. In each of these slices the tomogram is viewed as a $2 \mathrm{D}$ image, and segmentation is performed similar to 2D-TEM characterization of soot. The selected pixels from each slice are then combined into a 3D model of the particle that can be measured and viewed. In many instances, segmentation is carried out manually using imageprocessing software as low contrast, noise, and other reconstructions artefacts decrease image quality to the point that automated methods struggle [65]. Automated methods have been developed in many studies, though due to great variation in the appearance of tomograms that derive from TEM image characteristics, tilt-series composition, reconstruction methods, and the particular sample under study, automated methods that are generally applicable are yet to be developed [하].

It is well known that tomographic reconstruction from tilt-series data is subject to so-called missing wedge elongation artefacts [58]. This originates from the violation of the underlying Fourier Slice Theorem, that states that tomographic reconstruction requires all possible $2 \mathrm{D}$ projections to recreate the $3 \mathrm{D}$ object. In the case of tilt-series capture in TEM, this set of $2 \mathrm{D}$ projections is incomplete as the maximum tilt range that can be achieved is generally only $\pm 60-70^{\circ}$. This means that there is a lack of projection data of the particle taken from directions perpendicular to the TEM support film on which the particle is deposited (designated as the Z-direction). Missing Z-information in 2D propagates as an elongation in the $\mathrm{Z}$-direction in the resulting tomogram, and therefore the reconstructed particle. Clearly, this will cause reconstructed volumes to deviate from the true morphologies. The extent of this elongation in single-axis tomographic reconstruction is described by Radermacher's equation (2) [66], which provides a value of 1.55 for a single-axis $\pm 60^{\circ}$ tilt ( $55 \%$ stretch).

$$
e_{x z}=\sqrt{\frac{\alpha+\sin (\alpha) \cos (\alpha)}{\alpha-\sin (\alpha) \cos (\alpha)}}
$$

In some tomography studies, simulated data sets or model species are used to establish the accuracy of the reconstruction procedure $[\underline{64}, \underline{67}, \underline{68}, \underline{69}, \underline{70}]$. Okyay's SEM tomography study of soot used a simple sphere model, and observed no elongation for a $\pm 80^{\circ}$ reconstruction [64]. Our own study performed similar work and saw $15 \%$ Z-elongation for $\pm 60^{\circ}$ reconstruction of a sphere [ $\underline{63}$ ]. It is common for simplified structures to be used as model systems due to difficulties associated with the creation of synthetic data-sets. However, it is also accepted that the shape of the structure to be reconstructed has a defining effect on the extent of elongation observed [툐], so the relevance may be limited.

Electron tomography has been shown in a number of introductory studies to possess potential as a replacement for traditional 2D TEM characterization. However, little followup work has been carried out primarily due to the technique being too slow to be a viable alternative. Recent work by the authors has presented efforts towards improving the throughput of 3D-TEM [ 63] , though as yet due consideration has not been given to ensuring its accuracy. The purpose of this study is to quantify the accuracy of 3D-TEM, and in particular our optimized methodology. Simulated soot nanoparticles were created so that exact values for morphological parameters were known, and so that reconstruction artefacts such as missing-wedge elongation were realistically represented. These synthetic models were then subjected to a 3D-TEM procedure, and were characterized in terms of their morphology in order to compare to the known values.

\section{Experimental}

Using the image processing software Fiji [ㄴ6], 13 models were created to represent small soot nanoparticles, similar to those seen in our previous studies of GDI soot-in-oil samples. The models were made from attaching between 10 and 30 spheres (representing primary particles) that were 100 pixels in diameter. If real primary particles are usually around $25 \mathrm{~nm}$ in diameter, the size of these spheres represents a scale of 
roughly $0.25 \mathrm{~nm}_{\text {pixel }}{ }^{-1}$, which is towards the highest resolution of real tilt-series we have acquired experimentally. The aggregation algorithm was not intended to mimic real soot formation to a quantitative degree, merely to produce structures that qualitatively represented soot nanoparticles. The procedure is summarized as follows:

- A single sphere is positioned at the centre of the model

- A second sphere is attached to the first in a random position in $\mathrm{x}, \mathrm{y}, \mathrm{Z}$ between predefined limits of overlap $(10-20 \%)$

- One of the existing spheres is randomly chosen to act as the seed for the following sphere

- The new sphere is overlapped with the seed sphere in a randomly chosen position within the limits of overlap

- This process repeats until all spheres have been attached.

- If the same spheres are chosen as a seed multiple times, it results in a more clustered structure, whereas more branched structures result in the opposite scenario

This procedure produces models in the form of a binary 8 -bit *.tiff image stack. All white pixels correspond to the model, while black pixels represent background only. The true values of all morphological parameters (surface area, volume) can easily be measured in Fiji. These measurements are henceforth referred to as 'ground truth' or 'true' values. Figure 2 provides an example of 5 of the simulated soot models, rendered using UCSF Chimera [1]].

Original models are then subjected to a realistic tomographic reconstruction procedure in order to evaluate the extent and propagation of errors in 3D-TEM. Firstly, a TEM-style tilt-series of $2 \mathrm{D}$ projections is created from the original models using the volume viewer plugin for Fiji. Volume viewer allows the user to represent a 3D volume in 2 -dimensions from a chosen viewing angle. Tilt-series were created over a $\pm 80^{\circ}$ tilt range (in the y-direction) in $1^{\circ}$ increments. Volume viewer also allows for alteration of the contrast and transparency of the projections to replicate real TEM images and adhere to the projection requirement for tomography. Next, images are inverted and contrast is altered so that grey-levels in these simulated tilt-series match those seen in real TEM images of soot.

Typical grey-levels for were measured from 300 mesh Copper grids with lacey carbon support and graphene oxide

\section{FIGURE 2 \\ Simulated soot nanoparticles rendered in UCSF} Chimera [71]

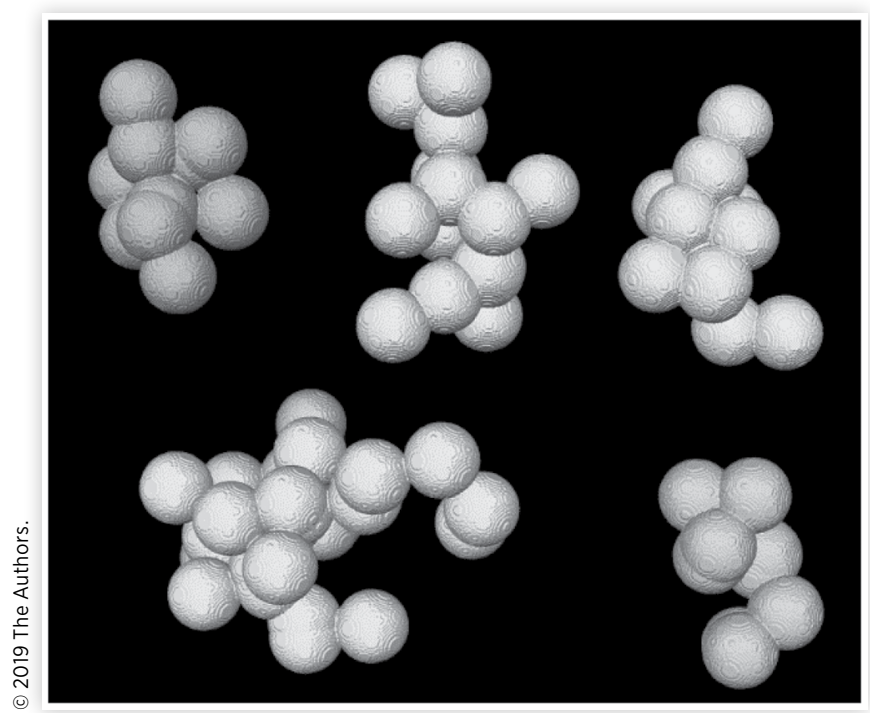

film, as has been used experimentally in our previous investigations. Average values for the graphene oxide (GO) film, and single/layered primary particles on GO were measured in real TEM images of a variety of soot samples.

Next, TEM stylings were applied to the tilt-series to visually match the noise and blurring artefacts that are present in real TEM images. The specified noise plugin was used to add random noise, and blurring was achieved using a subsequent smooth image function. At this point, three variations of each tilt-series were made for each model: the full $\pm 80^{\circ}$ tilt-series (161 images), a $\pm 60^{\circ}$ tilt-series (121 images), and a $\pm 60^{\circ}$ tilt-series with $3^{\circ}$ increments (41 images; henceforth referred to as $\left.\pm 60 \_3^{\circ}\right)$. These represent the maximum tilt series that can be reconstructed with the IMOD/Etomo software $\left( \pm 80^{\circ}\right)$, the largest tilt range that can routinely be recorded experimentally with TEM $\left( \pm 60^{\circ}\right)$, and the optimum tilt-series as per our previous publication $\left( \pm 60^{\circ}\right.$ with $3^{\circ}$ increments) [ $\underline{63}$ ]. Figure 3 shows an example of images from a simulated tilt-series

For each of the models, all 3 tilt-series were reconstructed and tomograms produced (39 reconstructions total). Reconstructions were performed using IMOD with Etomo [72], utilizing the weighted back-projection (WBP) algorithm, as per the optimized results of our previous publication [63].

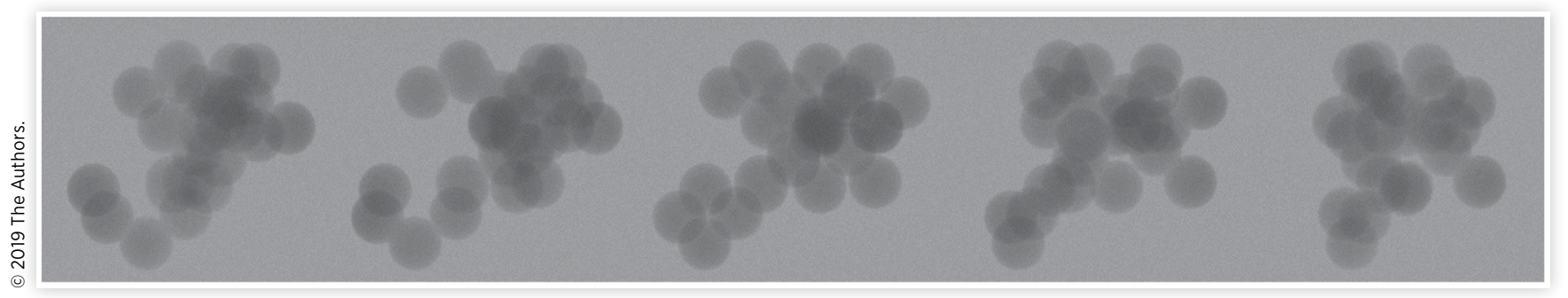




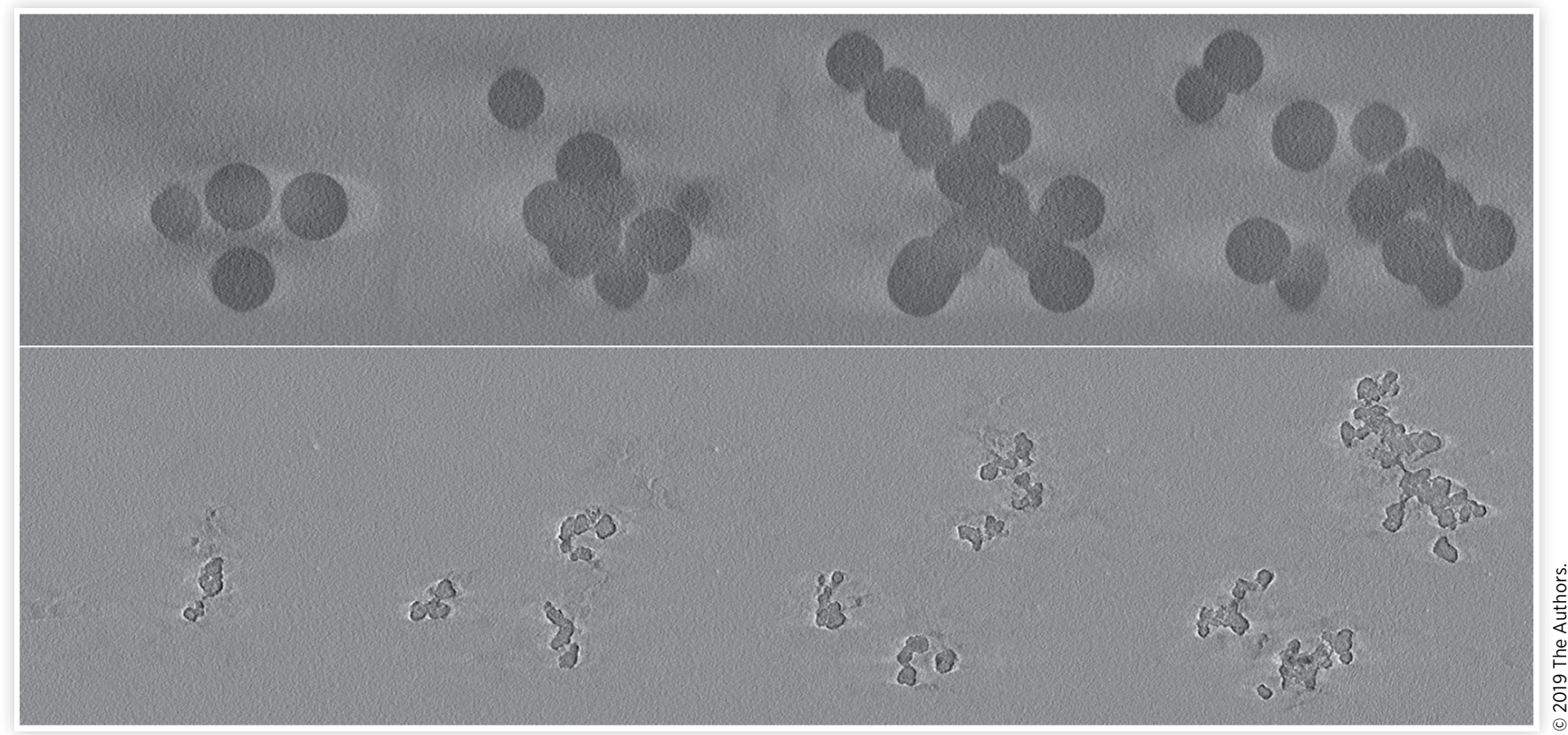

Reconstruction of the full $\pm 80^{\circ}$ tilt-series took around 1 minute to complete ( $30-50 \mathrm{mb}$ tilt-series file), $\pm 60^{\circ}$ tilt-series around 30 seconds $(20-40 \mathrm{mb})$, and $\pm 60 \_3^{\circ}$ around 10 seconds (5-15mb). Reconstructions were all performed on a Lenovo PC with a $3.50 \mathrm{GHz}$ Intel Xeon E5-1620v3 processor (8 CPUs) and 32 GB RAM, using a 64-bit version of Windows 10 .

For all 3 versions of reconstruction for each model, the maximum Z-length of the particle in the tomogram was measured to assess the extent of the missing-wedge elongation. This was performed manually and without any prior referral to true value in the original models to reduce risk of biased selections.

For all models, the $\pm 60 \_3^{\circ}$ tomograms were segmented via a process that employed use of manual selections and linear interpolation to varying degrees in an iterative process. It should be noted that the tomogram is a solid $3 \mathrm{D}$ volume, and segmentation is carried out by arranging this into $2 \mathrm{D}$ (1-voxel thick) slices in the Z-direction. First, every $20^{\text {th }}$ slice in the tomogram was manually segmented, i.e. all pixels in a slice relating to particle matter are distinguished from the background, using Fiji.

Segmentations of the intermediate slices are automatically generated using linear interpolation, and the resulting model is created and measured. Next, a model is created by manually segmenting every $10^{\text {th }}$ slice and interpolating intermediate results. As every $20^{\text {th }}$ slice has previously been segmented, half of the manual selections already exist. The remaining slices are corrected from those produced by the previous application of linear interpolation. This is then repeated between every $5^{\text {th }}$ slice of the tomogram.

Finally, every $2^{\text {nd }}$ slice is considered manually, and interpolation used to create a model that is considered to be fully manually segmented (interpolation between every $2^{\text {nd }}$ slice is generally excellent and effectively identical to manual segmentation of all slices, though much quicker to produce). Example images from simulated soot tomogram, and images from a real soot tomogram are shown in Figure 4.

As mentioned, this method cascades through increasingly-manual segmentations and makes use of the interpolated results from the previous iteration. As the proportion of manual segmentation increases, interpolated results are more accurate and less time and effort is required to correct selections manually when moving to the next level of interpolation (e.g. every $10^{\text {th }}$ to every $5^{\text {th }}$ slice). At the fully manual (every $2^{\text {nd }}$ slice) segmentation level, the majority of slices require no correction at all. This iterative method saves a significant amount of time in comparison to producing each separately, especially between the $5^{\text {th }}$ slice and fully manual interpolation levels. This segmentation procedure resulted in 4 different models being created for each $\pm 60 \_3^{\circ}$ tilt-series: $20^{\text {th }}$ slice with interpolation, $10^{\text {th }}$ slice with interpolation, $5^{\text {th }}$ slice with interpolation, and fully manual segmentation.

For 2 models only, the $\pm 80^{\circ}$ tomogram was fully segmented. This represents the most complete reconstruction that could be performed with Etomo, and is a larger tilt-range than is likely to be possible experimentally. The reconstruction is created from the greatest amount of $2 \mathrm{D}$ data, and should therefore be the highest quality of the three reconstructions produced for each model.

Morphological characterization of all the original and reconstructed models was carried out using Fiji and the BoneJ plugin [73]. Volume was measured exactly in terms of voxels. Surface area was measured with BoneJ by creating a mesh of triangular faces that define the shape of the object in 3D graphics (the default settings were used for all models). Radius gyration was calculated on a voxel basis assuming equal density of voxels, according to the equation (3), where $n_{v x}$ is the total number of voxels, and $r_{i}$ is the distance from each pixel to the centre of mass. A shape descriptor parameter similar to sphericity [74] was derived in order to define the 
overall morphology of the particles. Our parameter was derived on a similar basis to the 2D parameter circularity, which gives a value of 1 for a circle and goes towards 0 for $1 D$ line. For differentiating from sphericity, we termed our parameter '3D-circularity'. 3D-ciruclarity outputs a value of 1 for an object that is perfectly spherical, and moves towards 0 for objects that are highly 2-dimensional or extremely branched. Equation (4) shows the calculation for 3D-circularity, where $V$ is the volume of the structure, and $A_{s}$ is its surface area.

$$
\begin{gathered}
R_{g}=\sqrt{\frac{\sum_{i=1}^{n_{v x}} r_{i}^{2}}{n_{v x}}} \\
\text { Circ.3D }=6 \sqrt{\pi}\left(\frac{V}{A_{s}^{3 / 2}}\right)
\end{gathered}
$$

\section{Results \& Discussion}

In this work, 13 simulated soot-like models were created to act as ground truth for assessing the accuracy of an optimized 3D-TEM procedure. Values of morphological parameters are known exactly for the original models and are compared to results produced after simulated 2D-projections of the models are subjected to a realistic tomographic reconstruction procedure. The efforts taken to reflect genuine soot morphology and real tomographic reconstruction were made given that it is known, but not robustly described, that deviation from the true structure occurs during reconstruction and that it depends upon the morphology of the structure in question. Given the characteristically complex, fractal-like structure of soot nanoparticles and potential benefits offered by a novel 3D-TEM methodology, it was deemed that the use of a simple spherical model as ground truth would not be adequate.

\section{Missing Wedge Elongation}

For each of the 13 ground truth models, three tomograms were produced from tilt-series that varied in the number of images and total tilt range. In theory, larger tilt-range and greater number of images should result in a less elongated, more accurate tomogram. The tilt-series were positioned so that Z-depth in the tomogram matched that in the original models. As such, it was possible to quickly measure total Z-length of the particle in the tomogram by simply finding the first and last slices where particle matter appears. See Figure 4 for examples of tomogram images. All selections were made manually, and without prior referral to the ground truth value to limit biased selections. Results are shown in Table 1.

TABLE 1 Average elongation of thirteen reconstructed soot

\begin{tabular}{|c|c|c|c|}
\hline Tilt-Series & $\begin{array}{l}\text { Z-elongation } \\
\%\end{array}$ & $\begin{array}{l}\text { Z-elongation } \\
\text { No. slices }\end{array}$ & $\begin{array}{l}\text { Standard } \\
\text { Error }\end{array}$ \\
\hline $\pm 80^{\circ}$ & $0.4 \%$ & 1.3 & 0.13 \\
\hline $\pm 60^{\circ}$ & $4.0 \%$ & 12.8 & 0.28 \\
\hline $\pm 60 \_3^{\circ}$ & $3.5 \%$ & 11.5 & 0.23 \\
\hline
\end{tabular}
models vs ground truth Z-length
For the $\pm 80^{\circ}$ tilt-series (largest tilt-range than can be reconstructed with Etomo) elongation was marginal. Only 2 of the models showed elongation by more than a single slice (equivalent to $\sim 0.25 \mathrm{~nm}$ in the simulated scale), and on average elongation was $0.4 \%$ of the original ground truth Z-length. For $\pm 60^{\circ}$ tilt-series reconstruction, elongation was more pronounced at an average of $4.0 \%$ longer in $\mathrm{Z}$ than the original model. For the $\pm 60 \_3^{\circ}$ tilt-series, average elongation was marginally less than was seen for $\pm 60^{\circ}$ reconstructions, at $3.5 \%$ longer $v s$ ground truth. Though this was consistent for most of the models (7 out of the 13), the difference is small and the average elongations are well within standard error such that the degree of elongation for the two are equivalent.

These results show that elongation of soot tomograms due to the missing wedge is small, and much less than predicted by Radermacher's equation [66]. In comparison to our previous attempts using the reconstruction of a simple sphere model (15\% Z-elongation), this highlights the effect of the shape of the object on the extent of elongation. When converted to approximated real size using 1 pixel $=0.25 \mathrm{~nm}$, typical elongation for reconstructions of $\pm 60^{\circ}$ or $\pm 60 \_3^{\circ}$ tiltseries is around $3 \mathrm{~nm}$ for a soot aggregate.

\section{$\pm 80^{\circ}$ Reconstruction and Comparison to $\pm 60 \_3^{\circ}$}

For 2 of the soot-like models (named Random6 \& Random9), the $\pm 80^{\circ}$ tomograms were fully segmented. Previously, only the Z-lengths of the particles were measured from these tomograms. The $\pm 80^{\circ}$ tilt-series contains the largest range of $2 \mathrm{D}$ information that can be reconstructed with Etomo. Results were compared to those from the $\pm 60 \_3^{\circ}$ reconstructions to further understand the effect of tilt-series composition on the accuracy of reconstructions. Results are presented in Table 2 below, for full manual segmentation:

For both $\pm 80^{\circ}$ reconstructions measurements of volume, surface area, and radius of gyration are slightly underestimated in comparison to the ground-truth values. This underestimation is more pronounced in surface area, leading to slight increases in 3D-circularity values for both models $v s$ GT, indicating that the shape of the model differs slightly. In comparison to $\pm 80^{\circ}$ reconstruction, the $\pm 60 \_3^{\circ}$ method

TABLE 2 Morphological results from $\pm 80^{\circ}$ and $\pm 60 \_3^{\circ}$ reconstructions. Values shown are deviations from ground truth values for reconstructed models, as a percentage (positive results indicate values larger than $\mathrm{GT}$, and vice versa for negative). ' $\Delta \%$ ' shows the change between $\pm 80^{\circ}$ and $\pm 60 \_3^{\circ}$ results; e.g. positive values indicate $\pm 60 \_3^{\circ}$ results are an improvement on $\pm 80^{\circ}$ results $c f$. ground truth, and

\begin{tabular}{|c|c|c|c|c|c|c|}
\hline & & \multicolumn{5}{|c|}{ \% Deviation vs Ground Truth } \\
\hline & & $\mathbf{v}$ & $\mathbf{A}_{\mathbf{s}}$ & $\mathbf{R}_{\mathbf{g}}$ & 3D-Circ. & Z-length \\
\hline \multirow[t]{2}{*}{ Random6 } & $\pm 80^{\circ}$ & -1.1 & -2.0 & -0.3 & 2.1 & 0.3 \\
\hline & $\pm 60 \_3^{\circ}$ & 2.3 & -0.4 & 0.1 & 2.9 & 4.6 \\
\hline \multirow[t]{2}{*}{ Random9 } & $\pm 80^{\circ}$ & -0.9 & -1.2 & -0.3 & 0.9 & 0.4 \\
\hline & $\pm 60 \_3^{\circ}$ & 2.0 & 0.8 & -0.2 & 0.9 & 4.5 \\
\hline
\end{tabular}
vice versa. 
results in an increase in the absolute values of volume and surface area by area $2-3 \%$, and in radius of gyration by around $0.5 \%$. Despite causing a relative increase in the measured bulk of the particles (volume and surface area), the fact that the magnitude of the increase is small and that $\pm 80^{\circ}$ results are inherently underestimated means that $\pm 60 \_3^{\circ}$ reconstruction is similarly (and highly) accurate in comparison to GT.

\section{$\pm 60 \_3^{\circ}$ Reconstruction: Full Morphological Analysis}

In total, 13 reconstructions were performed on tilt-series captured over $\pm 60^{\circ}$ ranges with $3^{\circ}$ increments. This tilt-series configuration was found to be the optimum choice in terms of speed and quality in a previous publication [63]. In that work, in terms of accuracy it was found that overall tilt-range was more important than total number of images, and that $3^{\circ}$ increments significantly reduced potential beam exposure whilst having no significant negative effect on tomogram quality. However, 'acceptable' quality in that work was defined as providing results similar to those of $\pm 60^{\circ}$ reconstruction, which in theory should be more accurate. In the current work, we are able to compare results of $\pm 60 \_3^{\circ}$ reconstruction directly to the ground-truth values, and can provide a definitive answer on the quality of this protocol. Results are shown in Table 3 , and Figure 5 shows a visual comparison of an original simulated soot model, and the same model after $\pm 60 \_3^{\circ}$ reconstruction has been performed.

TABLE 3 Mean deviations in morphology for $\pm 60 \_3^{\circ}$ reconstructions of the thirteen simulated models.

\begin{tabular}{|c|c|c|c|c|}
\hline & Volume & $\begin{array}{l}\text { Surface } \\
\text { Area }\end{array}$ & $\begin{array}{l}\text { Radius of } \\
\text { Gyration }\end{array}$ & 3D-Circularity \\
\hline $\begin{array}{l}\text { Mean \% } \\
\text { Deviation from } \\
\text { GT }\end{array}$ & 2.0 & -0.1 & 0.2 & 2.3 \\
\hline $\begin{array}{l}\text { Max. \% } \\
\text { Deviation from } \\
\text { GT }\end{array}$ & 5.0 & 2.8 & 0.5 & 6.6 \\
\hline $\begin{array}{l}\text { Min. \% Deviation } \\
\text { from GT }\end{array}$ & 0.1 & -2.6 & -0.2 & -0.7 \\
\hline $\begin{array}{l}\text { Standard } \\
\text { Deviation }\end{array}$ & 1.70 & 1.71 & 0.21 & 2.40 \\
\hline
\end{tabular}

FIGURE 5 Comparison of the original (left in both images) 'random17' simulated particle, and the $\pm 60 \_3^{\circ}$ reconstructed particle (right in both). Both images are rendered in UCSF Chimera, viewing the $X Y$-plane (left) and YZ-plane (right).

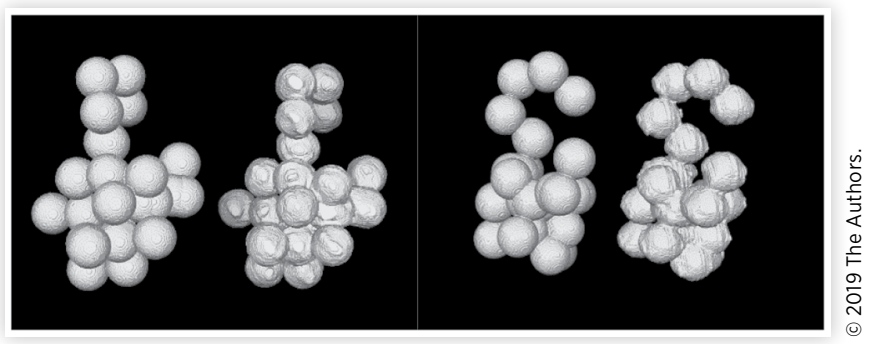

These results show that the reconstruction of the simulated soot-like models created for this investigation via the $\pm 60 \_3^{\circ}$ methodology is generally excellent. Important and descriptive morphological parameters show only minor deviations from ground-truth values on average, and even maximum deviations are well within $10 \%$. All values of volume were seen to be overestimated in comparison to ground truth, whereas surface area was split almost evenly between under- and over-estimation. Deviation in radius of gyration were generally marginal at most. $3 \mathrm{D}$-circularity is used to describe the shape of the particles, as it involves a ratio of volume to surface area. Deviations in 3D-circularity were predominantly increases in comparison to ground truth values. As 3D-circularity goes towards a value of 1 for more spherical/clustered objects, this indicates a change in the shape of reconstructed particles such that they are slightly bulkier and possess less rugged edges (increased volume, decreased surface area). There were no clear trends that linked the magnitude of deviations in 3D-circularity with any other morphological parameter (e.g. volume, surface area, radius of gyration, Z-length, absolute 3D-circularity, size, etc.).

If it can be accepted that the simulated soot nanoparticle reconstruction demonstrated in this work is a realistic representation of both genuine soot structures and reconstruction artefacts errors, then this data shows that 3D-TEM can produce highly accurate morphological measurements. This is important given the large deviations that have been observed between $2 \mathrm{D}$ - and 3D-derived morphological characterizations $[\underline{18}, \underline{25}, \underline{28}, \underline{53}]$, and the uncertainty that exists within $2 \mathrm{D}$-derived results [푸].

\section{Effect of Interpolation on Segmentation}

It was seen in our previous publication that the use of interpolation in segmentation could greatly improve the speed of 3D-TEM characterization, though can result in significant decreases in particle volume and surface area that greatly affect the accuracy of the model. The optimal method was $5^{\text {th }}$ slice interpolation, which on average required $40 \%$ less time than full manual segmentation, but was associated with average decreases in volume and surface area of 3.4 and $3.9 \%$ respectively. In the current work, the accuracy of interpolated results can be quantified in comparison to the ground truth values. It was decided that the $3 \mathrm{D}$-circularity parameter would be the best parameter to determine accuracy of reconstruction, as in some situations interpolation can result in e.g. an extremely accurate surface area, while volume is inaccurate. As $3 \mathrm{D}$-circularity takes into account both parameters it is more useful as a reflection of the trueness of the reconstructed particle volume. Results are shown in the Table 2 below.

These results indicate that both $5^{\text {th }}$ slice and $10^{\text {th }}$ slice interpolation produce models of similar quality to that of full manual segmentation. Despite producing the most accurate volume results, $20^{\text {th }}$ slice interpolation sees a drop-off in $3 \mathrm{D}$-circularity accuracy due to larger decreases in surface area. However, the results of $20^{\text {th }}$ slice interpolation are still reasonably accurate, and outperform the results in our previous publication. This is likely due to the fact that the scale 
TABLE 4 Average deviations from ground truth in terms of morphology at different levels of interpolation segmentation, for $\pm 60 \_3^{\circ}$ reconstructions

\begin{tabular}{|c|c|c|c|}
\hline & \multicolumn{3}{|c|}{ Average \% deviation vs GT } \\
\hline & Vol & SA & 3D-circ \\
\hline full & 2 & -0.1 & 2.3 \\
\hline 5th & 2.3 & 0.2 & 2 \\
\hline 10th & 2.5 & -0.3 & 2.9 \\
\hline 20th & 1.5 & -2.5 & 5.5 \\
\hline
\end{tabular}

of the simulated soot models in this study is towards the finer scales that would regularly be found in real TEM tilt-series. The previous study included results from tilt-series scaled at $\sim 0.3$ to $1.1 \mathrm{~nm}$ per pixel. The coarser the scale, the larger increments used for interpolated segmentation are in respect to the size of the whole tomogram. This means that while the finer details of the soot structure may be retained at $20^{\text {th }}$ slice interpolation at finer scales, at coarser scales 20 slices of the tomogram may be similar to the size of entire primary particles. As such, in some areas of the tomogram this can result in complete removal of finer details and subsequently large deviations from the true structure. As such, the accuracy of the interpolation methods may differ depending on the scale of the TEM images used.

\section{Conclusions}

This work highlights that 3D-TEM is a new high-fidelity tool for soot nanoparticle characterization, which is able to overcome the limitations of more traditional 2D-TEM methods. It permits direct measurement of morphological parameters such as volume, surface area, and radius of gyration of 3-dimensional soot structures, without the significant errors associated with orientation uncertainty and empirical correction factors of 2D-TEM. The optimized reconstruction procedure described in this and a previous paper [63] greatly improves the speed of 3D-TEM, allowing for its use for high-throughput soot characterization, and has now been shown to produce highly accurate 3D volume reconstructions. In particular, the work carried out on the simulated soot nanoparticles has led to the following conclusions:

- $\pm 80^{\circ}$ reconstruction results in marginal elongation only $(<1 \%)$

- $\pm 60^{\circ}$ and $\pm 60 \_3^{\circ}$ reconstruction result in minor elongation only (3-4\%)

- In comparison to $\pm 80^{\circ}$ reconstruction, $\pm 60 \_3^{\circ}$ method causes increase to bulk of the particle (surface area and volume) though inherent underestimation at $\pm 80^{\circ}$ means methods are similarly accurate

- $\pm 60 \_3^{\circ}$ reconstruction (full manual segmentation) in general is highly accurate, with average values of volume, surface area, radius of gyration, and 3D-circularity all within $3 \%$ of ground truth values
- Use of interpolation at $5^{\text {th }}$ and $10^{\text {th }}$ slice levels is similarly accurate to full manual segmentation, though at $20^{\text {th }}$ slice we see drop off in 3D-circ ( $>5 \%$ deviation)

- Previously only $5^{\text {th }}$ slice was acceptable, due to different scale of simulated models

\section{References}

1. Broday, D.M. and Rosenzweig, R., "Deposition of FractalLike Soot Aggregates in the Human Respiratory Tract," Journal of Aerosol Science 42(6):372-386, 2011, doi:10.1016/j. jaerosci.2011.03.001.

2. Yang, H.-M., Barger, M.W., Castranova, V., Ma, J.K. et al., "Effects of Diesel Exhaust Particles (DEP), Carbon Black, and Silica on Macrophage Responses to Lipopolysaccharide: Evidence of DEP Suppression of Macrophage Activity," Journal of Toxicology and Environmental Health Part A 58(5):261-278, 1999.

3. Løvik, M., Høgseth, A.-K., Gaarder, P.I., Hagemann, R. et al., "Diesel Exhaust Particles and Carbon Black Have Adjuvant Activity on the Local Lymph Node Response and Systemic IgE Production to Ovalbumin," Toxicology 121(2):165178, 1997.

4. Mari, X., Lefevre, J., Torreton, J.P., Bettarel, Y. et al., "Effects of Soot Deposition on Particle Dynamics and Microbial Processes in Marine Surface Waters," Global Biogeochemical Cycles 28(7):662-678, 2014, doi:10.1002/2014gb004878.

5. Koelmans, A.A., Jonker, M.T., Cornelissen, G., Bucheli, T.D. et al., "Black Carbon: The Reverse of Its Dark Side," Chemosphere 63(3):365-377, 2006.

6. Cattaneo, R., Rouviere, C., Rassoulzadegan, F., and Weinbauer, M.G., "Association of Marine Viral and Bacterial Communities with Reference Black Carbon Particles under Experimental Conditions: An Analysis with Scanning Electron, Epifluorescence and Confocal Laser Scanning Microscopy," FEMS Microbiology Ecology 74(2):382396, 2010.

7. Khalizov, A.F., Xue, H.X., Wang, L., Zheng, J. et al., "Enhanced Light Absorption and Scattering by Carbon Soot Aerosol Internally Mixed with Sulfuric Acid," Journal of Physical Chemistry A 113(6):1066-1074, 2009, doi:10.1021/ jp807531n.

8. Fan, J., Zhang, R., Tao, W.K., and Mohr, K.I., "Effects of Aerosol Optical Properties on Deep Convective Clouds and Radiative Forcing," Journal of Geophysical Research: Atmospheres 113(D8), 2008.

9. George, S., Balla, S., Gautam, V., and Gautam, M., "Effect of Diesel Soot on Lubricant Oil Viscosity," Tribology International 40(5):809-818, 2007, doi:10.1016/j. triboint.2006.08.002.

10. Ferraro, G., Fratini, E., Rausa, R., Fiaschi, P. et al., "Multiscale Characterization of Some Commercial Carbon Blacks and Diesel Engine Soot," Energy \& Fuels 30(11):98599866, 2016.

11. Apicella, B., Ciajolo, A., Tregrossi, A., Abrahamson, J. et al., "HRTEM and EELS Investigations of Flame-Formed Soot Nanostructure," Fuel 225:218-224, 2018. 
12. Wang, L., Song, C., Song, J., Lv, G. et al., "Aliphatic C-H and Oxygenated Surface Functional Groups of Diesel InCylinder Soot: Characterizations and Impact on Soot Oxidation Behavior," Proceedings of the Combustion Institute 34(2):3099-3106, 2013.

13. Apicella, B., Senneca, O., Russo, C., Heuer, S. et al., "Separation and Characterization of Carbonaceous Particulate (Soot and Char) Produced from Fast Pyrolysis of Coal in Inert and CO2 Atmospheres," Fuel 201:118-123, 2017.

14. Vander Wal, R.L. and Tomasek, A.J., "Soot Oxidation: Dependence upon Initial Nanostructure," Combustion and Flame 134(1-2):1-9, 2003.

15. Pfau, S., La Rocca, A., Haffner-Staton, E., Rance, G. et al., "Comparative Nanostructure Analysis of Gasoline Turbocharged Direct Injection and Diesel Soot-In-Oil with Carbon Black," Carbon 139:342-352, 2018.

16. Vander Wal, R.L. and Tomasek, A.J., "Soot Nanostructure: Dependence upon Synthesis Conditions," Combustion and Flame 136(1-2):129-140, 2004.

17. Kandas, A.W., Senel, I.G., Levendis, Y., and Sarofim, A.F., "Soot Surface Area Evolution during Air Oxidation as Evaluated by Small Angle X-Ray Scattering and CO2 Adsorption," Carbon 43(2):241-251, 2005, doi:10.1016/j. carbon.2004.08.028.

18. Adachi, K., Chung, S.H., Friedrich, H., and Buseck, P.R., "Fractal Parameters of Individual Soot Particles Determined Using Electron Tomography: Implications for Optical Properties," Journal of Geophysical Research-Atmospheres 112(D14), 2007, doi:Artn D1420210.1029/2006jd008296.

19. Harris, S.J. and Maricq, M.M., "Signature Size Distributions for Diesel and Gasoline Engine Exhaust Particulate Matter," Journal of Aerosol Science 32(6):749-764, 2001.

20. George, S., Balla, S., and Gautam, M., "Effect of Diesel Soot Contaminated Oil on Engine Wear," Wear 262(9-10):11131122, 2007, doi:10.1016/j.wear.2006.11.002.

21. Zhang, Q. and Rubini, P., "Modelling of Light Extinction by Soot Particles," Fire Safety Journal 46(3):96-103, 2011.

22. Kahnert, M. and Devasthale, A., "Black Carbon Fractal Morphology and Short-Wave Radiative Impact: A Modelling Study," Atmospheric Chemistry and Physics 11(22):1174511759, 2011.

23. Lapuerta, M., Oliva, F., and Martínez-Martínez, S., "Modeling of the Soot Accumulation in DPF under Typical Vehicle Operating Conditions," SAE Int. J. Fuels Lubr. 3(2):532-542, 2010, doi:10.4271/2010-01-2097.

24. La Rocca, A., Di Liberto, G., Shayler, P.J., and Fay, M.W., "The Nanostructure of Soot-In-Oil Particles and Agglomerates from an Automotive Diesel Engine," Tribology International 61:80-87, 2013, doi:10.1016/j.triboint.2012.12.002.

25. Martos, F.J., Lapuerta, M., Expósito, J.J., and SanmiguelRojas, E., "Overestimation of the Fractal Dimension from Projections of Soot Agglomerates," Powder Technology 311:528-536, 2017.

26. Clague, A.D.H., Donnet, J., Wang, T.K., and Peng, J.C.M., "A Comparison of Diesel Engine Soot with Carbon Black," Carbon 37(10):1553-1565, 1999, doi:10.1016/S00086223(99)00035-4.

27. Lapuerta, M., Martos, F.J., and Herreros, J.M., "Effect of Engine Operating Conditions on the Size of Primary
Particles Composing Diesel Soot Agglomerates," Journal of Aerosol Science 38(4):455-466, 2007, doi:10.1016/j. jaerosci.2007.02.001.

28. Rogak, S.N. and Flagan, R.C., "Characterization of the Structure of Agglomerate Particles," Particle \& Particle Systems Characterization 9(1):19-27, 1992, doi:10.1002/ ppsc.19920090104.

29. Koylu, U.O., Faeth, G.M., Farias, T.L., and Carvalho, M.G., "Fractal and Projected Structure Properties of Soot Aggregates," Combustion and Flame 100(4):621-633, 1995, doi:10.1016/0010-2180(94)00147-K.

30. Ishiguro, T., Takatori, Y., and Akihama, K., "Microstructure of Diesel Soot Particles Probed by Electron Microscopy: First Observation of Inner Core and Outer Shell," Combustion and Flame 108(1-2):231-234, 1997, doi:10.1016/S00102180(96)00206-4.

31. Uy, D., Ford, M.A., Jayne, D.T., O'Neill, A.E. et al., "Characterization of Gasoline Soot and Comparison to Diesel Soot: Morphology, Chemistry, and Wear," Tribology International 80:198-209, 2014, doi:10.1016/j. triboint.2014.06.009.

32. La Rocca, A., Bonatesta, F., Fay, M.W., and Campanella, F., "Characterisation of Soot in Oil from a Gasoline Direct Injection Engine Using Transmission Electron Microscopy," Tribology International 86:77-84, 2015, doi:10.1016/j. triboint.2015.01.025.

33. Vander Wal, R.L., Yezerets, A., Currier, N.W., Kim, D.H. et al., "HRTEM Study of Diesel Soot Collected from Diesel Particulate Filters," Carbon 45(1):70-77, 2007.

34. Van Gulijk, C., Marijnissen, J., Makkee, M., Moulijn, J. et al., "Measuring Diesel Soot with a Scanning Mobility Particle Sizer and an Electrical Low-Pressure Impactor: Performance Assessment with a Model for Fractal-Like Agglomerates," Journal of Aerosol Science 35(5):633-655, 2004.

35. Symonds, J.P., Reavell, K.S.J., Olfert, J.S., Campbell, B.W. et al., "Diesel Soot Mass Calculation in Real-Time with a Differential Mobility Spectrometer," Journal of Aerosol Science 38(1):52-68, 2007.

36. Gargiulo, V., Alfè, M., Di Blasio, G., and Beatrice, C., "Chemico-Physical Features of Soot Emitted from a DualFuel Ethanol-Diesel System,” Fuel 150:154-161, 2015.

37. La Rocca, A., Di Liberto, G., Shayler, P.J., Parmenter, C.D.J. et al., "Application of Nanoparticle Tracking Analysis Platform for the Measurement of Soot-In-Oil Agglomerates from Automotive Engines," Tribology International 70:142-147, 2014, doi:10.1016/j.triboint.2013.09.018.

38. Dobbins, R.A. and Megaridis, C.M., "Morphology of FlameGenerated Soot as Determined by Thermophoretic Sampling," Langmuir 3(2): 254-259, 1987, DOI10.1021/ $\underline{\text { la00074a019. }}$.

39. Yamaguchi, T., Kondo, K., Nishigai, H., Takano, S. et al., "Direct Sampling, TEM Analysis and Optical Measurement of Soot Particles at Different Axial Locations in a Transient Spray Flame," SAE Int. J. Fuels Lubr. 5(1):316-328, 2012, doi:10.4271/2011-01-2051.

40. Kook, S. and Pickett, L.M., "Soot Volume Fraction and Morphology of Conventional, Fischer-Tropsch, CoalDerived, and Surrogate Fuel at Diesel Conditions," SAE Int. J. Fuels Lubr. 5(2):647-664, 2012, doi:10.4271/2012-01-0678. 
41. Su, D.S., Jentoft, R.E., Müller, J.-O., Rothe, D. et al., "Microstructure and Oxidation Behaviour of Euro IV Diesel Engine Soot: A Comparative Study with Synthetic Model Soot Substances," Catalysis Today 90(1):127$132,2004$.

42. Lapuerta, M., Ballesteros, R., and Martos, F.J., "The Effect of Diesel Engine Conditions on the Size and Morphology of Soot Particles," International Journal of Vehicle Design 50(1-4):91-106, 2009.

43. Alfè, M., Apicella, B., Barbella, R., Rouzaud, J.-N. et al., "Structure-Property Relationship in Nanostructures of Young and Mature Soot in Premixed Flames," Proceedings of the Combustion Institute 32(1):697-704, 2009.

44. Brodowski, S., Amelung, W., Haumaier, L., Abetz, C. et al., "Morphological and Chemical Properties of Black Carbon in Physical Soil Fractions as Revealed by Scanning Electron Microscopy and Energy-Dispersive X-Ray Spectroscopy," Geoderma 128(1-2):116-129, 2005.

45. Fernandes, M.B., Skjemstad, J.O., Johnson, B.B., Wells, J.D. et al., "Characterization of Carbonaceous Combustion Residues. I. Morphological, Elemental and Spectroscopic Features," Chemosphere 51(8):785-795, 2003.

46. Schindelin, J., Arganda-Carreras, I., Frise, E., Kaynig, V. et al., "Fiji: An Open-Source Platform for Biological-Image Analysis," Nature Methods 9(7):676-682, 2012, doi:10.1038/ Nmeth.2019.

47. Neer, A. and Koylu, U.O., "Effect of Operating Conditions on the Size, Morphology, and Concentration of Submicrometer Particulates Emitted from a Diesel Engine," Combustion and Flame 146(1-2):142-154, 2006, doi:10.1016/j. combustflame.2006.04.003.

48. Bonczyk, P.A. and Hall, R.J., "Fractal Properties of Soot Agglomerates,” Langmuir 7(6):1274-1280, 1991.

49. Koylu, U.O., McEnally, C.S., Rosner, D.E., and Pfefferle, L.D., "Simultaneous Measurements of Soot Volume Fraction and Particle Size/Microstructure in Flames Using a Thermophoretic Sampling Technique," Combustion and Flame 110(4):494-507, 1997, doi:10.1016/S00102180(97)00089-8.

50. Gwaze, P., Schmid, O., Annegarn, H.J., Andreae, M.O. et al., "Comparison of Three Methods of Fractal Analysis Applied to Soot Aggregates from Wood Combustion," Journal of Aerosol Science 37(7):820-838, 2006, doi:10.1016/j. jaerosci.2005.06.007.

51. Lapuerta, M., Ballesteros, R., and Martos, F.J., "A Method to Determine the Fractal Dimension of Diesel Soot Agglomerates," Journal of Colloid and Interface Science 303(1):149-158, 2006, doi:10.1016/j.jcis.2006.07.066.

52. van Poppel, L.H., Friedrich, H., Spinsby, J., Chung, S.H. et al., "Electron Tomography of Nanoparticle Clusters: Implications for Atmospheric Lifetimes and Radiative Forcing of Soot," Geophysical Research Letters 32(24): 2005, DOI:Artn L2481110.1029/2005gl024461.

53. Orhan, O., Haffner-Staton, E., La Rocca, A., and Fay, M., "Characterisation of Flame-Generated Soot and Soot-In-Oil Using Electron Tomography Volume Reconstructions and Comparison with Traditional 2D-TEM Measurements," Tribology International 104:272-284, 2016, doi:10.1016/j. triboint.2016.09.015.
54. Medalia, A.I., "Morphology of Aggregates: VI. Effective Volume of Aggregates of Carbon Black from Electron Microscopy; Application to Vehicle Absorption and to Die Swell of Filled Rubber," Journal of Colloid and Interface Science 32(1):115-131, 1970.

55. Bardasz, E.A., Cowling, S.V., Ebeling, V.L., George, H.F. et al., "Understanding Soot Mediated Oil Thickening through Designed Experimentation-Part 1: Mack EM6-287, GM 6.2 L," SAE Technical Paper 952527, 1995, doi:10.4271/952527.

56. Adachi, K., Chung, S.H., and Buseck, P.R., "Shapes of Soot Aerosol Particles and Implications for Their Effects on Climate," Journal of Geophysical Research-Atmospheres 115, 2010, doi:Artn D1520610.1029/2009jd012868.

57. La Rocca, A., Campbell, J., Fay, M.W., and Orhan, O., "Soot-inOil 3D Volume Reconstruction Through the Use of Electron Tomography: An Introductory Study," Tribology Letters 61(1), 2016, doi:ARTN 810.1007/s11249-015-0625-Z.

58. Frank, J., Electron Tomography: Methods for ThreeDimensional Visualization of Structures in the Cell Second Edition (New York: Springer, 2008).

59. Midgley, P.A. and Weyland, M., "3D Electron Microscopy in the Physical Sciences: The Development of Z-Contrast and EFTEM Tomography," Ultramicroscopy 96(3-4):413-431, 2003, doi:10.1016/S0304-3991(03)00105-0.

60. Fernandez, J.J., "Computational Methods for Materials Characterization by Electron Tomography," Current Opinion in Solid State \& Materials Science 17(3):93-106, 2013, doi:10.1016/j.cossms.2013.03.002.

61. Batenburg, K.J., Fortes, W., Hajdu, L., and Tijdeman, R., "Bounds on the Difference between Reconstructions in Binary Tomography," Discrete Geometry for Computer Imagery 6607:369, 2011.

62. Batenburg, K.J., Bals, S., Sijbers, J., Kubel, C. et al., “3D Imaging of Nanomaterials by Discrete Tomography," Ultramicroscopy 109(6):730-740, 2009, doi:10.1016/j. ultramic.2009.01.009.

63. HAFFNER-STATON, E., La Rocca, A., and Fay, M.W., "Progress towards a Methodology for High Throughput 3D Reconstruction of Soot Nanoparticles via Electron Tomography," Journal of Microscopy 270(3):272-289, 2018.

64. Okyay, G., Heripre, E., Reiss, T., Haghi-Ashtiani, P. et al., "Soot Aggregate Complex Morphology: 3D Geometry Reconstruction by SEM Tomography Applied on Soot Issued from Propane Combustion," Journal of Aerosol Science 93:63-79, 2016, doi:10.1016/j.jaerosci.2015.11.009.

65. Fernandez, J.J., "Computational Methods for Electron Tomography," Micron 43(10):1010-1030, 2012, doi:10.1016/j. micron.2012.05.003.

66. Radermacher, M. and Hoppe W.. "Properties of 3-D Reconstruction from Projections by Conical Tilting Compared to Single-Axis Tilting," in Proceedings of the 7th European Congress on Electron Microscopy, 1980.

67. Friedrich, H., Guo, S., de Jongh, P.E., Pan, X. et al., "A Quantitative Electron Tomography Study of Ruthenium Particles on the Interior and Exterior Surfaces of Carbon Nanotubes," ChemSusChem 4(7): 957-963, 2011.

68. Ali, R.A., Landsberg, M.J., Knauth, E., Morgan, G.P. et al., "A 3D Image Filter for Parameter-Free Segmentation of 
Macromolecular Structures from Electron Tomograms," PLOS ONE 7(3):e33697, 2012, doi:10.1371/journal. pone.0033697.

69. Mairhofer, S., Sturrock, C., Wells, D.M., Bennett, M.J. et al., "On the Evaluation of Methods for the Recovery of Plant Root Systems from X-Ray Computed Tomography Images," Functional Plant Biology 42(5):460-470, 2015, doi:10.1071/ Fp14071.

70. Mezerji, H.H., Van den Broek, W., and Bals, S., "A Practical Method to Determine the Effective Resolution in Incoherent Experimental Electron Tomography," Ultramicroscopy 111(5):330-336, 2011.

71. Pettersen, E.F., Goddard, T.D., Huang, C.C., Couch, G.S. et al., "UCSF Chimera-A Visualization System for Exploratory Research and Analysis," J Comput Chem 25, 2004, doi:10.1002/jcc.20084.

72. Kremer, J.R., Mastronarde, D.N., and McIntosh, J.R., "Computer Visualization of Three-Dimensional Image Data Using IMOD," J Struct Biol 116, 1996, doi:10.1006/ jsbi.1996.0013.

73. Doube, M., Klosowski, M.M., Arganda-Carreras, I., Cordelieres, F.P. et al., "BoneJ Free and Extensible Bone Image Analysis in ImageJ,” Bone 47(6):1076-1079, 2010, doi:10.1016/j.bone.2010.08.023.

74. Wadell, H., "Sphericity and Roundness of Rock Particles," The Journal of Geology 41(3):310-331, 1933.

\section{Contact Information}

Correspondence to: Ephraim Haffner-Staton, Powertrain Research Group, Department of Mechanical Materials and Manufacturing Engineering, The University of Nottingham, Nottingham, NG7 2RD U.K. Tel: +44 (0)1159513872 ephraim.haffner-staton@nottingham.ac.uk

\section{Acknowledgments}

This work was supported by the Engineering and Physical Sciences Research Council [grant number EP/M506588/1], through the scholarship provided by EPSRC DTG Centre in Complex Systems and Processes for E. Haffner-Staton.

\section{Definitions/Abbreviations}

$\mathbf{A}_{\text {eff }}$ - Projection 2D area of soot nanoparticle $\mathbf{A}_{\mathbf{p p}}$ - Projected 2D area of primary particle

$\mathbf{A}_{\mathrm{s}}$ - Surface Area

$\mathbf{D}_{\mathrm{f}}$ - Fractal Dimension

$\mathbf{e}_{\mathbf{x z}}-$ Z-elongation factor

$\mathbf{k}_{\mathbf{a}}$ - Fractal prefactor

$\mathbf{N}_{\mathbf{p}}$ - Number of Primary Particles

$\mathbf{n}_{\mathbf{p x}}$ - Number of pixels

$\mathbf{r}_{\mathbf{i}}$ - Distance from pixel $i$ to centre of mass

$\mathbf{R}_{\mathbf{g}}$ - Radius of Gyration

V - Volume

3D-circ. - 3D-circularity: $\operatorname{Circ}_{\cdot 3 D}=6 \sqrt{\pi}\left(\frac{V}{A_{s}^{3 / 2}}\right)$
a - Fractal exponent (eq. 1)

Maximum tilt-angle (eq. 2)

DLS - Dynamic Light Scattering

GDI - Gasoline Direct Injection

ICE - Internal Combustion Engine

NTA - Nanoparticle Tracking Analysis

SEM - Scanning Electron Microscopy

SIRT - Simultaneous Iterative Reconstruction Technique

TEM - Transmission Electron Microscopy

WBP - Weighted Back-Projection

(c) 2019 The Authors. Published by SAE International. This Open Access article is published under the terms of the Creative Commons Attribution License (http:// creativecommons.org/licenses/by/4.0/), which permits distribution, and reproduction in any medium, provided that the original author(s) and the source are credited. 